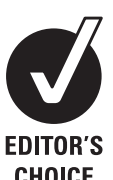

CHOICE
Pharmacology and Therapeutics Group, Department of Cardiovascular Sciences, University of Leicester, Clinical Sciences Building, Leicester Royal Infirmary, UK

\section{Correspondence to}

Dr lain Squire, Pharmacology and Therapeutics Group, Department of Cardiovascular Sciences, Clinical Sciences Building, Leicester Royal Infirmary LE2 7LX, UK;

is11@le.ac.uk

Accepted 25 March 2010

\title{
Identification of potential outcome benefit from ACE inhibition after acute coronary syndrome: a biomarker approach using $\mathrm{N}$-terminal proBNP
}

\author{
lain Squire, Paulene Quinn, Hafid Narayan, Sohail Khan, Onkar Dhillon, Kuan Ng, \\ Dominic Kelly, Joan Davies, Leong Ng
}

\section{ABSTRACT}

Objective To consider whether patients most likely to benefit from ACE inhibition in routine practice after acute coronary syndrome (ACS) may be identified from plasma natriuretic peptide concentrations.

Design Observational cohort study.

Setting Teaching hospital coronary care unit.

Patients 1725 patients admitted with acute coronary syndrome (56.3\% ST elevation ACS; median age 67, range 24-97 years).

Measurements Using Cox proportional hazards analysis, we assessed the adjusted predictive value for major adverse cardiac events (MACE) of prescription of an ACE inhibitor, of plasma N-terminal pro B-type natriuretic peptide (NT-proBNP) and for interaction between these factors. To adjust for demographic differences between patients prescribed or not prescribed an ACE inhibitor, a factor correcting for likelihood of ACE inhibitor prescription (propensity score) was included in the analysis.

Outcome measures The primary end point was the occurrence of MACE (death, recurrent myocardial infarction or hospitalisation with heart failure).

Results During the index admission ACE inhibitor was prescribed for 1267/1725 (73.4\%) patients. During follow-up (median 528 days, range $0-3873$ days), 534/1725 patients experienced MACE. After covariable adjustment, NT-proBNP showed linear association with risk of MACE $(p<0.005)$, strongest for patients with NT-proBNP in the top quartile of observed values $(H R=2.768, p<0.001)$. Only for patients with NT-proBNP in the top quartile was prescription of ACE inhibitor associated with reduction in risk of MACE $(H R=0.532, p=0.003)$. This association was maintained after correction for propensity scores ( $H R=0.599$, $\mathrm{p}=0.003$ ).

Conclusions Prognostic benefit from ACE inhibition was seen only in patients with the most marked elevation of plasma NT-proBNP. Plasma NT-proBNP may be a useful indicator of the appropriateness of individual prescription of ACE inhibitor treatment across the spectrum of ACS.

\section{INTRODUCTION}

Contemporary management of patients with acute myocardial infarction (AMI), involves the use of complex pharmacological treatment. This practice is based upon the results of large-scale randomised controlled trials (RCTs), demonstrating survival benefit associated with thrombolysis, ${ }^{1}$ and secondary prevention treatments, including inhibitors of platelet aggregation, ${ }^{2} \beta$ blockers ${ }^{3}$ and statins, ${ }^{4}$ started soon after the index event.

Several clinical RCTs ${ }^{5-10}$ demonstrated survival and symptomatic benefit associated with prescription of inhibitors of angiotensin converting enzyme (ACE) after AMI. The benefits of these agents have been consistent in those trials recruiting patients at high risk of adverse outcome after AMI, ${ }^{5-7} 10$ while in less selected populations the benefit of these agents has been less clear. ${ }^{8} 911$

In view of this background it has been argued that after AMI, ACE inhibitors should be prescribed to all patients in the immediate post-MI period but continued long term only in those with impaired left ventricular function. ${ }^{12}$ Moreover, in contrast to an earlier study, ${ }^{13}$ a later placebo-controlled trial ${ }^{14}$ indicated no benefit of ACE inhibition in patients with stable coronary disease and preserved left ventricular ejection fraction, calling in to question the routine prescription of ACE inhibitors in patients with coronary artery disease. Nonetheless, European and North American guidelines recommend consideration of ACE inhibition for all patients with both ST elevation ${ }^{15} 16$ and non STelevation ${ }^{17}{ }^{18}$ events, and in the UK, national guidelines recommend the prescription of ACE inhibitor treatment to all patients after AMI. ${ }^{19}$

After acute coronary syndrome (ACS), plasma concentrations of B-type natriuretic peptide (BNP) 2021 and the amino terminal fragment of its precursor, NTproBNP, 223 provide powerful prognostic information. Importantly, for patients with relatively low circulating natriuretic peptide levels in the first days after ACS, prognosis is relatively good..$^{20-23}$ No clinical trial after AMI or ACS has randomised patients to active or placebo treatment based upon natriuretic peptide concentrations. We wished to assess whether circulating NT-proBNP concentrations may usefully identify those patients for whom prescription of ACE inhibitor treatment confers, or alternatively does not confer, clinical benefit.

\section{PATIENTS AND METHODS}

\section{Study design and patient population}

Patients were recruited to a single-centre study of the association with outcome after ACS of plasma biomarkers including NT-proBNP. We recruited patients to University Hospitals of Leicester (catchment population 924000 in the 2001 census) between 1 March 2000 and 31 July 2007 with a diagnosis of ACS admitted, the criteria for which were appropriate symptoms, in the presence of 
dynamic ST-T changes and/or elevation of cardiac biomarkers. We categorised ACS in to ST elevation AMI (STEMI) or non-ST elevation AMI (NSTEMI). We excluded patients normally resident outside our health authority area, and those unwilling or unable to provide written consent. For patients with multiple admissions with ACS during the study period, only the first presentation was considered. From patient charts, we recorded the highest Killip class, ECG data and pharmacological treatment prescribed during the index admission, together with details of medical history, including previous AMI, angina, hypertension and diabetes.

For the current analysis, prescription of angiotensin receptor blocker (ARB) was considered equivalent to prescription of ACE inhibitor. As this was an observational cohort study, we reduced potential imbalance between ACE inhibitor/ARB-treated and non-treated patients using propensity score analysis. The prescription/non-prescription of ACEI or ARB was made by the doctor responsible for the care of each individual patient, blind to patient participation in the study. Our unit uses the UK National guidelines, ${ }^{19}$ which indicate that management should involve routine prescription of ACE inhibitor, antiplatelet treatment, statin and $\beta$ blocker. The study was approved by the hospital ethical review board and eligible patients gave written informed consent before inclusion. The authors had full access to the data and take responsibility for its integrity. All authors have read and agree to the manuscript as written.

\section{End points}

The prespecified primary end point was the combination of first major adverse cardiovascular event (MACE), defined as all-cause death, recurrent AMI or hospitalisation with heart failure.

Recurrent MI was defined as hospitalisation with a final discharge diagnosis of AMI, based upon the criteria according to the index admission. Hospitalisation with heart failure was defined as hospital admission for which the primary reason was considered to be heart failure, based upon presentation with appropriate signs and symptoms and requiring high dose oral or intravenous diuretic treatment, or inotropic support.

All deaths were identified through information supplied routinely to our hospital from the Office of National Statistics. Hospitalisations were identified through our hospital electronic patient tracking system with full review of all case records for verification of the date and cause of admission. Follow-up was censored at 1 August 2008, giving a minimum of 1-year's followup in all cases.

\section{Blood sampling and NT-proBNP assay}

Venous blood samples were obtained from convalescent patients, approximately $4-5$ days after symptom onset. Plasma was stored at $-80^{\circ} \mathrm{C}$ until assayed in batches. The NT-proBNP assay has been described previously. ${ }^{22}$ The lower limit of detection was $0.3 \mathrm{pmol} / 1$.

\section{Statistical analysis}

We examined the relationships with outcome of a variety of demographic, biochemical, clinical and therapeutic parameters. The predictive value of candidate variables was examined using univariable analysis and all factors showing univariable association with outcome at $p<0.10$ were then entered in multivariable analysis. Factors with non-Gaussian distribution were log-transformed before analysis.

Analysis was initially carried out including in the model $\mathrm{N}$-terminal proBNP as a continuous variable, and then divided into quartiles (quartile 4 highest), facilitating visualisation of the relationship with outcome, and assessment of any potential interaction with prescription/non-prescription of ACE inhibitor/ ARB. Finally, analysis was carried out including a term for interaction between NT-proBNP as a continuous variable $\times$ $\mathrm{ACEI} / \mathrm{ARB}$ or NT-proBNP quartile $\times \mathrm{ACEI} / \mathrm{ARB}$, with other variables within the base model, including ACEI/ARB treatment and NT-proBNP (as a continuous variable or quartile). The assigned treatment was that recorded as prescribed on discharge from hospital.

Differences among groups were examined using the Mann-Whitney $U$ test or Kruskal-Wallis test for continuous variables and Fisher exact test for categorical variables. Kaplan-Meier survival curves were compared using the log-rank test. Cox proportional hazards regression analysis was used to investigate the adjusted prognostic value associated with prescription of ACE inhibitor, of NT-proBNP quartile and for any potential interaction between these two factors. As this process indicated a statistically significant interaction between ACE inhibitor prescription and NT-proBNP quartile, we then calculated the adjusted hazard ratio for MACE associated with ACE inhibition, stratified by quartile of NT-proBNP and carried out sensitivity analyses to look for evidence of interaction between ACE inhibitor prescription and NT-proBNP in subgroups.

Two-tailed $p$ values were considered significant at $p<0.05$. Continuous data are presented as median and range. Analyses were carried out using SPSS version 16

\section{Propensity analysis}

We carried out a propensity analysis to adjust for differing characteristics between patients who received ACE inhibitor/ $A R B$ and those who did not. The propensity score (probability of being prescribed ACE inhibitor/ARB) was derived from a logistic model, containing the following variables: age; sex; maximum Killip class; type of MI (STEMI or NSTEMI); site of MI (anterior or other); estimated glomerular filtration rate; creatine kinase concentration; troponin I concentration; history of prior angina or MI, hypertension, diabetes or heart failure; in-patient prescription of thrombolytic treatment, aspirin, diuretic agent, calcium channel blocker, $\beta$ blocker, nitrate or statin.

Propensity scores were calculated using the above variables from 1565 patients (1197 were prescribed, and 368 not prescribed ACE inhibitor /ARB) for whom no data were missing. Survival analysis using Cox models was repeated on this population, entering the same variables as before and stratified for quintile of propensity score of the probability of ACE inhibitor/ARB prescription.

\section{RESULTS}

The study population consisted of 1725 (1241, 71.9\% male) patients recruited between 1 March 2000 and 31 July 2007. Of these, 971 (56.3\%) were classified as STEMI, and $754(43.7 \%)$ as NSTEMI. The mean age of patients was 66 years (median 67, range 24-97 years). Prior history of AMI or angina was recorded for 349 and 416 patients, respectively, with one or other condition recorded for $610(35.4 \%)$ patients. Previous hypertension was recorded in $820(47.5 \%)$ and diabetes in $393(22.8 \%)$ patients.

During the index hospitalisation, prescription of antiplatelet treatment occurred for 1502 (87.1\%) patients, $\beta$ blocker for 1359 (78.8\%), ACE inhibitor/ARB for 1267 (73.4\%), statin for 1319 (76.5\%), calcium channel blocker in $287(16.6 \%)$ and diuretic for 541 (31.4\%). Of 971 patients classified as presenting with 
STEMI, $646(66.5 \%)$ received reperfusion treatment with thrombolysis.

\section{N-terminal proBNP}

Plasma NT-proBNP (median $786.3 \mathrm{pmol} / \mathrm{l}$; interquartile (IO) range $254.0-2237.4 \mathrm{pmol} / \mathrm{l}$ ) was higher after STEMI (median 1010.4, IQ range 339.2-2681.8 $\mathrm{pmol} / \mathrm{l}$ ) than after NSTEMI (558.5, range IQ range $172.1-1770.5 \mathrm{pmol} / \mathrm{l}, \mathrm{p}<0.001)$, and in an anterior site (914.8, IQ range 295.4-2395.1 pmol/l) compared with other sites (672.5, IQ range $242.6-2154.3 \mathrm{pmol} / \mathrm{l}, \mathrm{p}=0.002$ ) of AMI.

Table 1 shows the characteristics of the population divided by NT-proBNP quartile. With increasing NT-proBNP quartile, the median age of patients, and the proportion of women, increased. Progressively higher NT-proBNP quartile was also associated with increasing proportions of STEMI, of anterior AMI, and of clinical evidence of heart failure. Higher NT-proBNP was also associated with greater comorbidity, with lower estimated glomerular filtration rate and greater prevalence of previous coronary heart disease (CHD), hypertension and diabetes.

\section{ACE inhibitor/ARB prescription}

Patients who received an ACE inhibitor or ARB were more likely to have had STEMI, or anterior AMI, and to have clinical evidence of heart failure (table 1). Peak concentrations of CK and troponin I were higher, and concomitant diabetes or hypertension more prevalent, in these patients. While the rate of administration of thrombolytic treatment was similar for patients prescribed or not prescribed ACE inhibitor/ARB, concomitant prescription of all other evidence-based secondary prevention treatments was more common with ACE inhibitor/ ARB prescription as was the frequency of in-hospital revascularisation (percutaneous coronary intervention or coronary artery bypass graft surgery).

\section{N-terminal proBNP and ACE inhibitor/ARB treatment: association with prognosis}

For patients alive at the end of the study, median follow-up was 585 days (range 131-3873). During follow-up, 534 individual patients $(31 \%)$ experienced a MACE. Two hundred and ninetyfour patients $(17 \%)$ died, 209 (12.1\%) experienced recurrent AMI, and 151 (8.8\%) experienced hospital admission for heart failure. As expected, risk of MACE increased by approximately $5 \%$ for each year of age. Lower estimated glomerular filtration rate, higher admission glucose, higher maximum Killip class and prior CHD, diabetes or hypertension were each associated with greater risk (table 2). Rate of MACE increased exponentially from quartile 1 to quartile 4 of NT-proBNP. In-hospital revascularisation and troponin-I levels were not independent predictors of MACE in these models (table 2).

\section{Interaction of NT-proBNP with ACE inhibitor/ARB treatment}

We observed strong, univariable association with MACE of NTproBNP, considered as a continuous variable and when divided by quartile (table 3 ). Cox proportional hazards modelling and Kaplan Meier survival analysis indicated that the survival benefit of ACEI/ARB was confined to those patients with NTproBNP elevated to the top quartile ( $\mathrm{HR}=0.613,95 \%$ CI 0.459 to $30.818, p=0.001$ (figure 1 ), with no statistically significant benefit in any other quartile (quartile $1 \mathrm{HR}=1.496$, $95 \%$ CI 0.870 to $2.572, p=0.146$; quartile $2 \mathrm{HR}=1.414,95 \%$ CI 0.846 to $2.364, p=0.187$; quartile $3 \mathrm{HR}=0.736,95 \%$ CI 0.500 to $1.082, p=0.119) 4$ or in quartiles $1-3$ combined $(p=0.241)$.

\section{Sensitivity analyses}

We further looked for an interaction between survival benefit from ACE inhibition and NT-proBNP concentration in subgroups of interest. Significant interaction was apparent for type of AMI (STEMI/NSTEMI), Killip class $(>1 / 1)$, left ventricular ejection fraction (LVEF; $\geq 40 \% /<40 \%$ ), history of diabetes and history of hypertension. ACE inhibition was associated with survival benefit in patients with STEMI (HR=0.599, $95 \%$ CI 0.402 to $0.895, \mathrm{p}=0.012$ ), but not in NSTEMI ( $\mathrm{HR}=0.786,95 \%$ CI 0.501 to $1.231, \mathrm{p}=0.293$ ); in those with Killip class $>1$ (HR $=0.613,95 \%$ CI 0.381 to $0.986, p=0.04)$ and also those with Killip class of 1 ( $\mathrm{HR}=0.558,95 \%$ CI 0.362 to $0.859, \mathrm{p}=0.008)$; in those with LVEF $<40 \%$ (HR $=0.343,95 \% \mathrm{CI}$ 0.155 to $0.761, p=0.008$ ), but not in those with LVEF $\geq 40 \%$ $(\mathrm{HR}=0.733,95 \%$ CI 0.435 to $1.233, \mathrm{p}=0.241)$.

Interestingly, ACE inhibitor treatment was associated with survival benefit in those patients without a history of diabetes ( $\mathrm{HR}=0.595,95 \% \mathrm{CI} 0.419$ to $0.813, \mathrm{p}=0.004$ ) or of hypertension $(\mathrm{HR}=0.588,95 \% \mathrm{CI} 0.393$ to $0.879, \mathrm{p}=0.010)$ but not in patients with prior history of these conditions (diabetes, $\mathrm{HR}=0.879,95 \% \mathrm{CI} 0.472$ to $1.639, \mathrm{p}=0.685$; hypertension, $\mathrm{HR}=0.700,95 \%$ CI 0.442 to $1.109, \mathrm{p}=0.129)$.

\section{Secondary end points}

Similar findings were obtained when we assessed the interaction between NT-proBNP and ACE inhibitor in the prediction of secondary end points. For the combination of death or heart failure, prescription of ACE inhibitor/RB (adjusted $\mathrm{HR}=1.811$, $95 \%$ CI 1.307 to $2.509, \mathrm{p}<0.001)$ and NT-proBNP quartile ( $\mathrm{HR}=1.406,95 \%$ CI 1.125 to $1.757, \mathrm{p}=0.003$ ), were each associated with increased risk of adverse outcome. Once again there was interaction between ACE inhibitor/ARB prescription and the top quartile NT-proBNP, this combination being associated with reduced risk ( $\mathrm{HR}=0.498,95 \% \mathrm{CI} 0.309$ to 0.803 , $\mathrm{p}=0.004)$ compared with ACE inhibitor/ARB prescription in the remainder of the population. When we considered death alone as the end point, ACE inhibitor/ARB prescription remained associated with increased risk ( $\mathrm{HR}=1.809,95 \%$ CI 1.257 to 2.604, $\mathrm{p}=0.001)$, as did NT-proBNP quartile (1.388, 1.068-1.804, $p=0.014)$. The interaction of ACE inhibitor/ARB prescription with top quartile of NT-proBNP was associated with a statistically non-significant reduction in risk of death $(\mathrm{HR}=0.710$, $0.418-1.205, p=0.204)$.

\section{Propensity score analysis}

In order to balance the covariates that differed between the ACE/ARB-treated and untreated subgroups, we calculated the conditional probabilities of being prescribed these drugs. Propensity scores were calculated for 1565 patients with no missing data, 1197 of whom were, and 368 were not, prescribed ACE inhibitor/ARB. Stratified Cox proportional hazard modelling was then repeated, including variables as before with the addition of a quintile of propensity score. Receiver operating characteristic curve (ROC) analysis of the propensity score for $\mathrm{ACE} / \mathrm{ARB}$ treatment for prediction of actual treatment showed reasonable discrimination (ROC AUC (area under the curve) of 0.755 (95\% CI 0.727 to $0.782, p<0.0005)$. Comparison of observed and expected outcomes over deciles of risk in ACE/ARB treated or untreated patients indicated reasonable calibration (Hosmer and Lemeshow statistic $\chi^{2}=14.4, p=N S$ ). In the echocardiography subgroup, the inclusion of LVEF did not improve the discrimination of the logistic model for prediction of ACE/ARB treatment (ROC AUC 0.733 with LVEF vs 0.726 


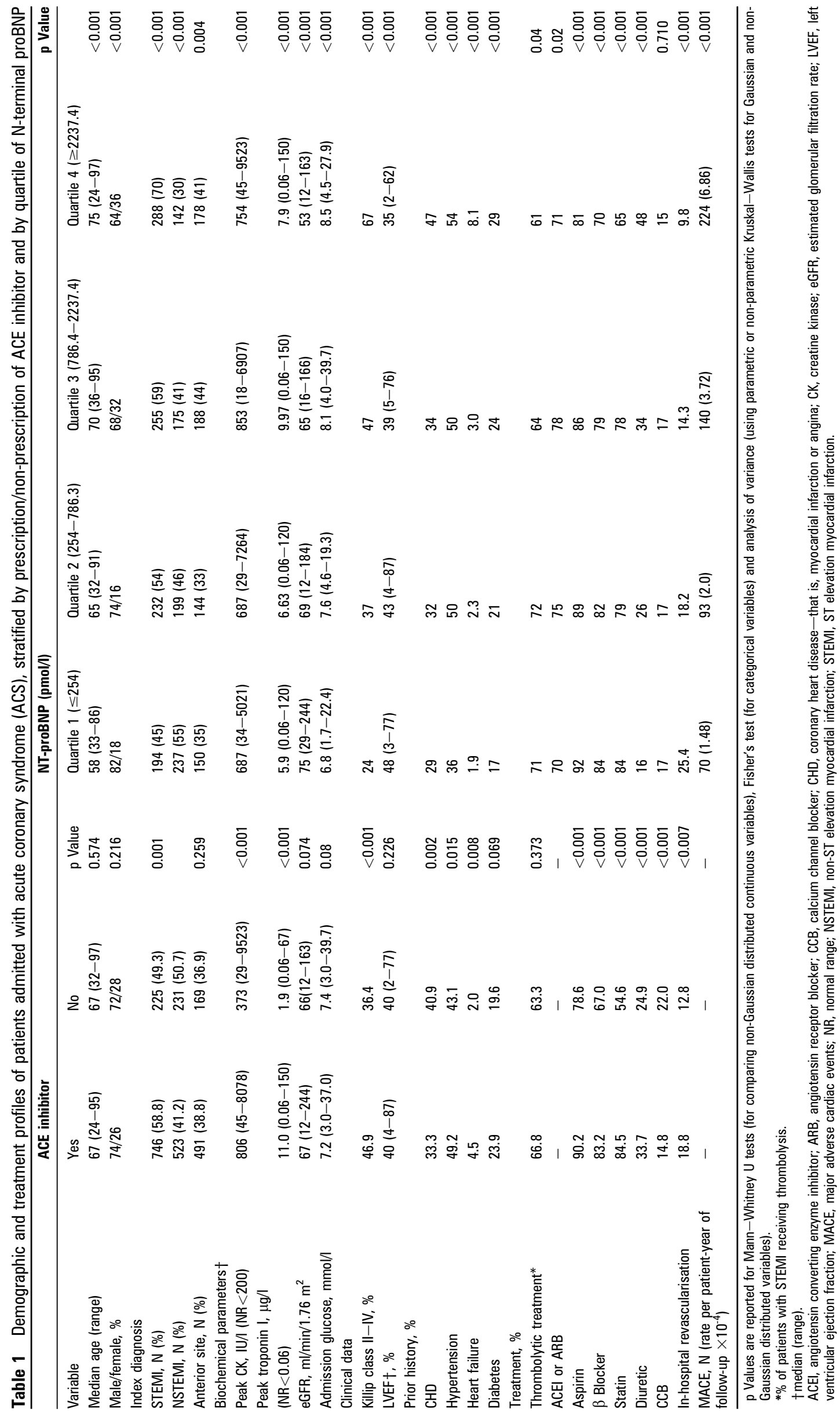


Table 2 Prediction of major adverse cardiovascular events (MACE): univariable and multivariable analysis

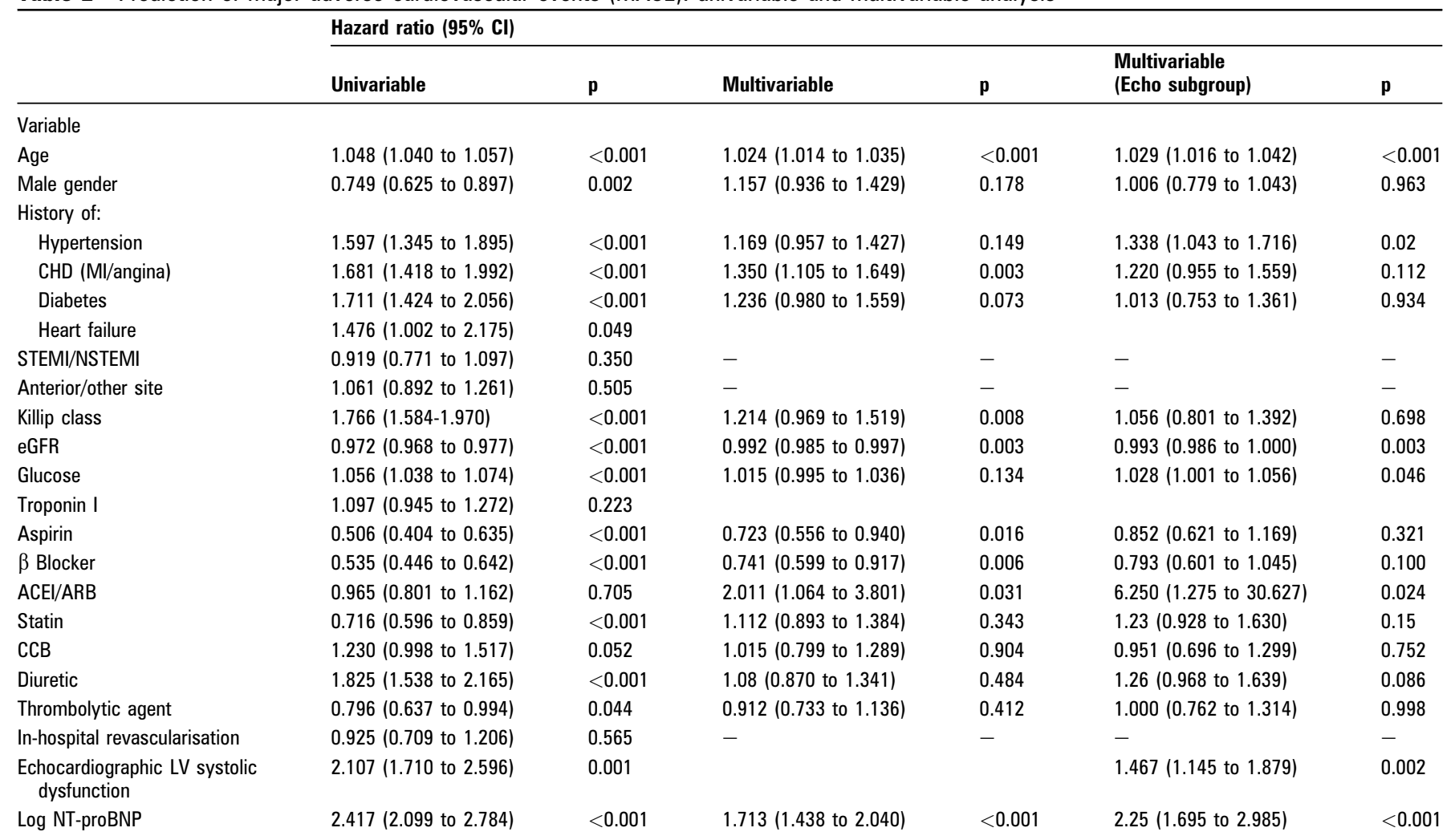

Multivariable analysis was carried out first including log NT-proBNP as a continuous variable, then substituting data categorised by quartile of NT- proBNP, which was finally replaced by a term for interaction between prescription of ACEI/ARB and quartile of NT-proBNP (quartile 4 compared with quartiles 1-3).

$\mathrm{ACEl}$, angiotensin converting enzyme inhibitor; $\mathrm{ARB}$, angiotensin receptor blocker; CCB, calcium channel blocker; CHD, coronary heart disease-that is, myocardial infarction or angina; CK, creatine kinase; eGFR, estimated glomerular filtration rate; MI, myocardial infarction; NSTEMI, non-ST elevation myocardial infarction; STEMI, ST elevation myocardial infarction.

without LVEF) nor was the ejection fraction a significant predictor of $A C E / A R B$ treatment.

Survival analysis corrected for quintile of propensity scores for probability of $\mathrm{ACE} / \mathrm{ARB}$ treatment confirmed the interaction between ACE inhibitor prescription and NT-proBNP, whether expressed as a continuous variable, in quartiles or dichotomised to the top quartile compared with quartiles 1-3. For NTproBNP expressed as a continuous variable or as NT-proBNP quartiles, the ACE/ARB prescription: NT-proBNP interaction was significant (NT-proBNP as continuous variable, $\mathrm{HR}=0.617$, $95 \%$ CI 0.447 to $0.852, p=0.003$; NT-proBNP as quartiles $\mathrm{HR}=0.744,95 \%$ CI 0.609 to $0.909, \mathrm{p}=0.004)$. For patients with NT-proBNP in the top quartile, compared with all other patients, prescription of ACE inhibitor remained associated with approximately $40 \%$ reduction in risk of MACE (HR $=0.574,95 \%$ $\mathrm{CI} 0.382$ to 0.862 ). For the end point of death or heart failure, the interaction remained statistically significant $(\mathrm{HR}=0.572,95 \% \mathrm{CI}$ 0.36 to $0.91, \mathrm{p}=0.018$ ). For death alone, the interaction term was not statistically significant ( $\mathrm{HR}=0.793,95 \%$ CI 0.474 to 1.328 , $\mathrm{p}=0.38)$.

These observations were not replicated for other secondary prevention treatments-namely, antiplatelet treatment, $\beta$ blockers or statins, for which there was no evidence of interactions with natriuretic peptide concentrations (data not shown).

\section{DISCUSSION}

To our knowledge this is the first study to consider whether in routine practice plasma natriuretic peptide concentration after ACS is indicative of the likely clinical benefit from ACE inhibition (or ARB). After adjustment for important clinical covariables, ACE inhibitor treatment is associated with survival benefit only for those patients with greatest elevation of plasma

Table 3 Adjusted hazard ratio (HR) for risk of major adverse cardiovascular events (MACE) in patients prescribed, compared with those not prescribed, ACE inhibitor, stratified by NT-proBNP concentration

\begin{tabular}{|c|c|c|c|c|}
\hline \multicolumn{5}{|l|}{ Hazard ratio $(95 \% \mathrm{Cl})$} \\
\hline & Univariable & p Value & Multivariable & p Value \\
\hline NT-proBNP quartile* & 2.417 (2.099 to 2.784 ) & $<0.001$ & 1.843 (1.401 to 2.424$)$ & $<0.001$ \\
\hline Top quartile NT-proBNP & $0.613(0.459$ to 0.818$)$ & 0.001 & 0.597 (0.439 to 0.813$)$ & 0.001 \\
\hline Quartiles 1-3 NT-proBNP & $1.174(0.898$ to 1.535$)$ & 0.241 & $1.114(0.845$ to 1.468$)$ & 0.443 \\
\hline Quartile 1 & $1.496(0.870$ to 2.572$)$ & 0.146 & 1.231 (0.687 to 2.203$)$ & 0.485 \\
\hline Quartile 2 & 1.414 (0.846 to 2.364$)$ & 0.187 & 1.341 (0.796 to 2.257 ) & 0.270 \\
\hline Quartile 3 & $0.736(0.500$ to 1.082$)$ & 0.119 & $0.938(0.624$ to 1.410$)$ & 0.758 \\
\hline
\end{tabular}

*NT-proBNP quartile considered as continuous variable.

HR was first calculated for NT-proBNP considered as a continuous variable. After demonstration of an interaction between NT-proBNP quartile and prescription of ACE inhibitor, HR for MACE in those prescribed an ACE inhibitor was calculated for quartile 4 of NT-proBNP, for quartiles 1-3, and for each quartile of NT-proBNP. 
Figure 1 Kaplan-Meier survival curves in patients prescribed (solid line) or not prescribed (broken line) ACE inhibitor/ ARB stratified by quartile of NT-proBNP concentration.
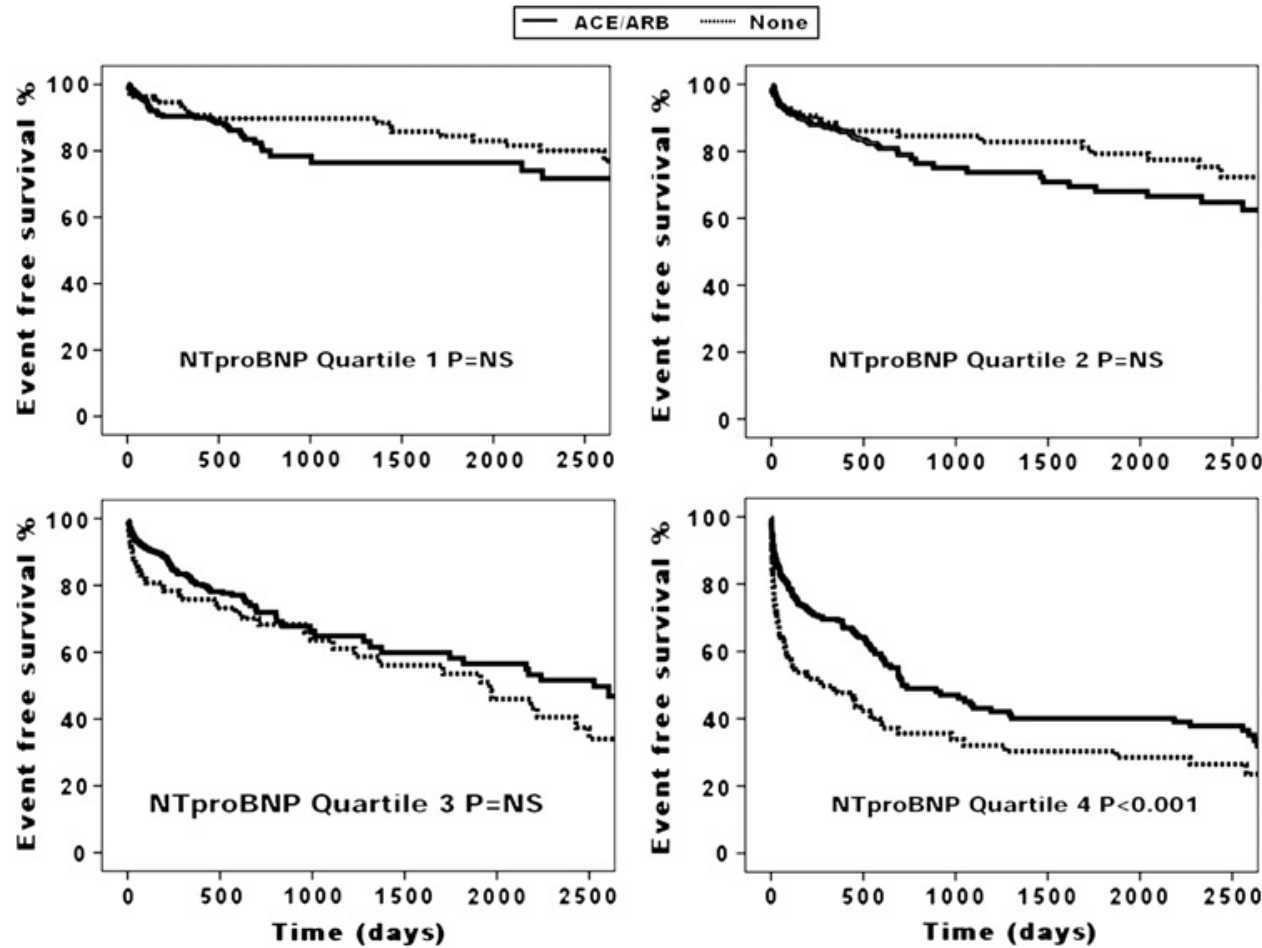

NT-proBNP. This association was maintained after Cox models were stratified according to propensity scores of the likelihood of being prescribed ACE inhibitor treatment and importantly, is seen in patients with NSTEMI as well as in those with STEMI.

The place of ACE inhibitors in CHD was initially established in populations with $\mathrm{AMI}^{5-10}$ and later investigated in lowerrisk populations with stable coronary artery disease and with preserved left ventricular function. ${ }^{13}{ }^{14}$ In these latter studies, ${ }^{13} 14$ the impact of ACE inhibition has been variable, with one showing survival benefit, ${ }^{13}$ and a second showing no impact upon outcome ${ }^{14}$ perhaps owing to lower cardiovascular risk in the latter population. ${ }^{14}$ Indeed, even among clinical trials in relatively high-risk populations after AMI, the impact of ACE inhibition has differed widely. ${ }^{5-10}$ The clearest, most consistent evidence of benefit after AMI was seen in studies of selected cohorts of patients with heart failure or LV dysfunction, ${ }^{5-7}$ in whom ACE inhibition was associated with absolute survival benefit of $4.2-7.6 \%$, compared with $0.3-1.8 \%$ in unselected cohorts. ${ }^{8-11}$ These observations support the concept that there may be a lower limit of risk below which ACE inhibition may confer no benefit.

However, while routine ACE inhibitor prescription after AMI may expose many to inappropriate treatment, greater harm may result from the failure to identify patients who are likely to benefit. ${ }^{12}$ Such concerns have contributed to recommendations that ACE inhibition should be considered for all patients after AMI ${ }^{15-18}$ However, there would be clear advantage in improved ability to individualise both treatment and prognosis after ACS, and a number of biomarkers have been investigated in this context. Of these, consistent prognostic utility has been shown for post-event plasma concentrations of B-type natriuretic peptides, over and above information from traditional risk factors. ${ }^{20-23}$ We now present data suggesting that plasma NT-proBNP may usefully identify cohorts of patients likely to obtain survival benefit from a specific drug treatment after ACS. We present clear evidence of interaction between plasma NTproBNP concentration and benefit from ACE inhibitor treatment, with event-free survival benefit being apparent only for those patients in whom plasma NT-proBNP concentration was elevated to above the top quartile of the population range. This observation held true even when adjusted for a variety of factors traditionally associated with adverse outcome, including concomitant secondary prevention drugs, and after stratifying models for propensity scores of probability of ACE inhibitor/ ARB treatment.

Further, this observation remained statistically robust when the analysis was confined to subgroups at greatest risk of adverse outcome, in whom survival benefit from ACE inhibition might be expected to be greatest. Thus, our observation of greater survival benefit from ACE inhibition in patients with markedly elevated NT-proBNP who had experienced STEMI, or who had LVEF $<40 \%$ lends strength to our overall findings. The lack of interaction between elevation in NT-proBNP and prior diagnosis of either diabetes or hypertension suggests that these parameters provide little prognostic information after AMI relative to that obtained from consideration of objective measures of infarct severity.

It has been suggested recently that there is a need for individualisation of treatment for cardiovascular disease 'beyond broadly based pharmacological therapies'. ${ }^{24}$ Our study suggests that after ACS, circulating levels of $\mathrm{N}$-terminal proBNP may be useful in this context.

Our observations contrast with the apparent lack of interaction between prescription of ACE inhibitor and plasma NTproBNP in a subcohort of the PEACE trial. ${ }^{25}$ While PEACE has the advantage of randomised controlled design, we suggest the apparent difference in conclusion may reflect the much lower cardiovascular risk of patients in PEACE compared with those in our report. Indeed, median plasma NT-proBNP in the PEACE substudy $^{25}$ was $139 \mathrm{pg} / \mathrm{ml}$, equivalent to $135 \mathrm{pmol} / \mathrm{l}$, and corresponding to quartile 1 in the current report. We suggest that the observations from PEACE are in fact consistent with our own and emphasise the potential for marked elevation of natriuretic peptide concentration as a marker of likely benefit from ACE inhibition in patients with cardiovascular disease. 


\section{Study limitations}

Our findings are limited by the observational nature of the study. While we adjusted extensively for covariables, and stratified by propensity scores, we are unable to rule out residual confounding by unknown covariables. While confounding by indication may be relevant, this is likely to have occurred irrespective of NT-pro BNP concentration. Indeed, demographic differences were more marked within quartiles 1-3 of NTproBNP: patients prescribed ACE inhibitor had higher peak CK, and had higher prevalence of anterior site of ACS, prior MI, concomitant hypertension and diabetes. In view of this background, the lack of survival benefit from ACE inhibition in these patients is even more striking. Our study may be criticised for being from a single centre, although from two hospitals. While our findings relate to NT-proBNP concentrations as observed in this specific population, this is countered by the unselected nature of our patients, representative of populations of patients with ACS in routine clinical practice, and the large number of patients included and of events observed. Finally, ACE inhibitor/ $\mathrm{ARB}$ usage was categorised according to prescription at discharge, and we have no data on the influence of, duration of, or changes in, treatment thereafter.

While an RCT based upon NT-proBNP concentration will be required to verify our observations, we suggest that without observational data such as those presented in this report, such a study could not be justified. In particular, no data have been reported from any randomised placebo controlled trial of ACE inhibitors after MI regarding the possibility of an interaction between outcome benefit and biomarker concentration. Our study is hypothesis generating and provides the basis for future prospective studies to assess the utility of natriuretic peptide concentrations as a marker of the likelihood of benefit from ACE inhibition after ACS. Our observations may have particular relevance in emerging economies, allowing limited healthcare resources to be used more cost effectively, against a background of increasing incidence of coronary artery disease in these countries.

In summary, we report an interaction between plasma NTproBNP after ACS with prescription of ACE inhibitor/ARB treatment. Only patients with the most marked elevation of plasma NT-proBNP showed outcome benefit from ACE inhibitor/ARB treatment. Plasma NT-proBNP may provide a useful indicator of the appropriateness of individual prescription of ACE inhibitor/ARB treatment across the spectrum of ACS.

Funding OD, SK and HN were supported by British Heart Foundation Junior Research Fellowships (grant numbers FS/03/028/15486, FS/03/028/15486 and FS/09/040, respectively) and LN and IS by the Leicester National Institute for Health Research Cardiovascular Biomedical Research Unit.

\section{Competing interests None.}

Ethics approval This study was conducted with the approval of the Leicestershire LREC.

Provenance and peer review Not commissioned; externally peer reviewed.

\section{REFERENCES}

1. Fibrinolytic Therapy Trialists (FTT) Collaborative Group. Indications for fibrinolytic therapy in suspected acute myocardial infarction: collaborative overview of early mortality and major morbidity results from all randomised trials of more than 1000 patients. Lancet 1994;343:311-22.

2. Antithrombotic Trialists Collaboration. Collaborative meta-analysis of randomised trials of antiplatelet therapy for prevention of death, myocardial infarction, and stroke in high risk patients. BMJ 2002;324:71-86.

3. Freemantle $\mathbf{N}$, Cleland $J$, Young $P$, et al. $\beta$ blockade after myocardial infarction: systematic review and meta regression analysis. BMJ 1999;318:1730-7.

4. Newby LK, Kristiansson A, Bhapkar MV, et al. Early statin initiation and outcomes in patients with acute coronary syndromes. JAMA 2002:287:3087-95.

5. Pfeffer M, Braunwald E, Moye LA, et al. Effect of captopril on mortality and morbidity in patients with left ventricular dysfunction after myocardial infarction. N Engl J Med 1992;327:669-77.

6. The Acute Infarction Ramipril Efficacy (AIRE) Investigators. Effect of ramipri on mortality and morbidity of survivors of acute myocardial infarction with clinical evidence of heart failure. Lancet 1993;342:821-8.

7. Kober L, Torp-Pedersen C, Carlsen JE, et al. A clinical trial of the angiotensin converting enzyme inhibitor trandolapril in patients with left ventricular dysfunction after myocardial infarction. N Engl J Med 1995;333:1670-6.

8. GISSI-3. Effects of lisinopril and transdermal glyceryl trinitrate singly and together on a 6 week mortality and ventricular function after acute myocardial infarction. Lancet 1994;343:1115-22

9. ISIS-4 Collaborative Group. ISIS-4: a randomised factorial trial assessing early oral captopril, oral mononitrate, and intravenous magnesium sulphate in 58050 patients with suspected acute myocardial infarction. Lancet 1995;345;669-85.

10. Ambrosioni E, Borghi C, Magnani B. The effect of the angiotensin-converting enzyme inhibitor zofenopril on mortality and morbidity after anterior myocardial infarction. N Engl J Med 1995;332:80-5.

11. Chinese Cardiac Study. Oral captopril versus placebo among 13,634 patients with suspected acute myocardial infarction: interim report from the Chinese Cardiac Study (CCS-1). Lancet 1995;345:686-7.

12. Cleland JGF. ACE inhibitors for myocardial infarction how should they be used? Eur Heart J 1995;16:153-9.

13. Fox KM. Efficacy of perindopril in reduction of cardiovascular events among patients with stable coronary artery disease: randomised, double-blind, placebo-controlled, multicentre trial (the EUROPA study). Lancet 2003;362:782-8.

14. The PEACE trial investigators. Angiotensin converting enzyme inhibition in stable coronary artery disease. N Engl J Med 2004;351:2058-68.

15. Van de Werf F, Ardissino D, Betriu A, et al. Management of acute myocardial infarction in patients presenting with ST-segment elevation. The task force on the management of acute myocardial infarction of the European Society of Cardiology. Eur Heart J 2003;24:28-66.

16. Antman E, Anbe DT, Armstrong PW, et al. Management of patients with STEMI; Executive Summary. J Am Coll Cardiol 2004:44:671-719.

17. The task force for the diagnosis and treatment of non ST segment elevation acute coronary syndromes of the European Society of Cardiology. Guidelines for the diagnosis and treatment of non ST segment elevation acute coronary syndromes. Eur Heart J 2007;28:1598-660.

18. Braunwald E, Antman EM, Beasley JW, et al. American College of Cardiology; American Heart Association. Committee on the management of patients with unstable angina. ACC/AHA 2002 guideline update for the management of patients with unstable angina and non-ST-segment elevation myocardial infarction-summary article: a report of the American College of Cardiology/American Heart Association task force on practice guidelines (Committee on the Management of Patients With Unstable Angina). J Am Coll Cardiol 2002;40:1366-74.

19. National Institute for Health and Clinical Excellence. Clinical guideline 48: MI secondary prevention: NICE 2007.

20. Omland T, Aakvaag A, Bonarjee VV, et al. Plasma brain natriuretic peptide as an indicator of left ventricular systolic function and long-term survival after acute myocardial infarction. Comparison with plasma atrial natriuretic peptide and $\mathrm{N}$-terminal proatrial natriuretic peptide. Circulation 1996;93:1963-9.

21. de Lemos JA, Morrow DA, Bentley JH, et al. The prognostic value of B-type natriuretic peptide in patients with acute coronary syndromes. $N$ Engl J Med 2001;345:1014-21.

22. Omland T, Persson A, Ng L, et al. N-terminal pro-B-type natriuretic peptide and longterm mortality in acute coronary syndromes. Circulation 2002;106:2913-18.

23. James SK, Lindahl B, Siegbahn A, et al. N-terminal pro-brain natriuretic peptide and othe risk markers for the separate prediction of mortality and subsequent myocardial infarction in patients with unstable coronary artery disease: a Global Utilization of Strategies to Open Occluded Arteries (GUSTO)-IV substudy. Circulation 2003;108:275-81.

24. Udelson JE. The inaugural issue of circulation heart failure. Circulation Heart Failure 2008;1:1

25. Omland T, Sabatine MS, Jablonski JA, et al. Prognostic value of B-type natriuretic peptides in patients with stable coronary artery disease. The PEACE trial. J Am Coll Cardiol 2007;50:205-14. 